\title{
Geociêncciass
}

\section{Classificação internacional do carvão (CEE-NU) da Camada Inferior, Mina do Leão, RS, Brasil}

\author{
Application of the international in-seam \\ coal classification for the Camada Inferior, \\ Leão Mine, RS, Brazil
}

Maristela Bagatin Silva

Professora, Dra.,

Engenharia Geológica

Engenharia de Petróleo

Centro de Desenvolvimento Tecnológico - CDTec

Universidade Federal de Pelotas - UFPEL

maristelabs@terra.com.br

\section{Resumo}

A aplicação de normas internacionais na classificação de carvões sul-brasileiros tem sido alvo de ampla discussão por causa da dificuldade de se obter uma perfeita correlação entre os diferentes parâmetros empregados, sobretudo na relação entre o poder refletor da vitrinita com outros parâmetros químicos, como o carbono e a matéria volátil. A Comissão Econômica para a Europa das Nações Unidas (CEE-NU) desenvolveu normas criteriosas para a classificação de carvões, tendo como base o uso simultâneo das três principais características dos carvões: composição, rank e grade, que devem ser consideradas simultaneamente, incluindo, também, análises suplementares como a determinação do poder calorífico superior, da umidade total da camada, do índice de inchamento (FSI) e análise elementar. Com a finalidade de se classificar o carvão da principal camada atualmente explotada na Mina do Leão pelas normas aprovadas pela CEE-NU, amostras foram coletadas e submetidas às análises petrográficas, imediatas, da umidade total e do poder calorífico superior. As camadas de carvão ocorrem na Formação Rio Bonito, Permiano (Artinskiano-Kunguriano), da bacia do Paraná. Com base nos resultados, o carvão da Camada Inferior do Leão pode ser classificado como carvão parabetuminoso (ou de grau médio D), conforme normas da Classificação Internacional da CEE-NU. Os resultados foram ainda comparados com dados de trabalhos anteriores com enfoque na avaliação do rank do carvão.

Palavras-chave: Carvão, classificação, Mina do Leão, Bacia do Paraná.

\begin{abstract}
The use of international standards for the classification of Southern Brazilian coal has shown wide variation in the determination of the real coal rank. The Economic Commission for Europe (ECE UN) has developed standard criteria for coal classification, based on the simultaneous consideration of its three main characteristics: composition, rank and grade. Supplementary analyses are also required. Samples from the main coal seam exploited in Leão, located in Rio Grande do Sul, Southeast Paraná Basin, were subjected to petrographic, proximate, bed moisture and calorific value determination, in order to be classified according to the UN ECE rules. The coal seams occur in the Rio Bonito, Permian (Artinskian-Kungurian) of the Paraná Basin. According to the International Classification (ECE-UN) the in-seam coals at Leão are in the parabetuminous coal range (or medium-rank coal D). The results were also compared with data from earlier studies focusing on aspects of coal rank.
\end{abstract}

Keywords: Coal, International In-seam Classification, Leão Mine, Paraná Basin. 


\section{Introdução}

Carvão é uma rocha sedimentar orgânica formada por uma mistura heterogênea de materiais orgânicos (macerais) e minerais (ICCP, 1963-1971). Sua classificação, nos estágios de carbonificação (rank), de linhito até antracito, é feita por meio de normas de classificação, que se valem de uma série de parâmetros fisicos, químicos e físico-químicos. Entretanto a classificação do carvão em categorias é difícil porque existem características que afetam a qualidade do carvão e, consequentemente, na sua classificação. Além disso, o valor comercial e a aplicação tecnológica do carvão podem ser afetados por uma classificação equivocada. No caso dos carvões sul-brasileiros, a classificação do carvão, segundo o rank, continua sendo alvo de ampla discussão, por causa das dificuldades da aplicação de normas internacionais de classificação. Os sistemas são até menos efetivos quando os parâmetros de rank são parâmetros químicos ou quando a influência da composição petrográfica não é considerada. Está também reconhecido que uma avaliação acurada dos depósitos

\section{Geologia}

A área de estudo faz parte da bacia do Paraná, que consiste de uma ampla região sedimentar do continente sul-americano, totalizando uma área que se aproxima dos 1,5 milhão de quilômetros quadrados. Os recursos de carvão in situ da Jazida do Leão são da ordem de $2.442 \times 10^{6} \mathrm{t}$, sendo $2.432 \times 10^{6} \mathrm{t}$ de recursos subterrâneos (DNPM/CPRM, 1986), e a camada Inferior é a principal camada de carvão explotada, na Mina do Leão (Figura 1).

Milani (1994) reconheceu, no re-

\section{Material e métodos}

Uma amostragem de cerca de $1 \mathrm{~kg}$ de carvão da Camada Inferior foi feita na frente mineira, seguindo os critérios estabelecidos na Norma ASTM D 1412 (CEE-NU,

\section{Análises químicas e físico-químicas}

As análises imediatas, incluindo a determinação da umidade para análise, cinzas e matéria volátil, foram realizadas conforme normas SABS 924, ISO 1171 e ISO 562 (CEE-NU, 1998), sendo que de carvão requer ainda a definição clara das técnicas de amostragem. Com base nessas considerações, a Comissão Econômica para a Europa das Nações Unidas (CEE-NU, 1998) publicou, no final da década de noventa, a Classificação Internacional do Carvão em Camada com o propósito de criar um instrumento que pudesse contribuir para a caracterização dos depósitos do carvão sem fins ou tratados de cunho comercial. De acordo com a metodologia adotada na classificação do carvão em camada, a sistematização do carvão deve ser baseada em três características fundamentais, as quais devem, sempre, ser utilizadas em seu conjunto. Essas características incluem o grau de carbonificação (rank), a composição petrográfica e a categoria ou grade (grau de impurezas). Os parâmetros de rank e da composição petrográfica são baseados nos resultados das análises petrográficas: valor médio do poder refletor aleatório da vitrinita $(\mathrm{Ra} \%)$ e grupos de macerais, sem matéria mineral, respectivamente. $\mathrm{O}$ poder calorífico superior, recalculado em base úmida, sem cinzas

gistro estratigráfico da bacia do Paraná, seis Supersequências (OrdovicianoSiluriano ao Neocretáceo). A sequência deposicional do carvão da Formação Rio Bonito está localizada na base da sequência de terceira ordem de Milani et al. (1994), a qual consiste de uma espessa sequência sedimentar da bacia (até $2.800 \mathrm{~m}$ ). Essa sequência compreende às unidades litoestratigráficas Itararé, Rio Bonito, Palermo e Irati (base). A Formação Rio Bonito é representada por uma sucessão sedimentar cíclica de pacotes de arenitos, siltitos e folhe-

1998) e encaminhada para análises de umidade total da camada, poder calorífico e elementar, no Laboratório da Faculdade de Ciências do Porto, Portugal. A seguir, ou-

o percentual de carbono fixo foi obtido por diferença. A umidade total e o poder calorífico superior foram determinados segundo as normas ISO 5068 e ISO 1928 (CEE-NU, 1998), respectivamente.
$(\mathrm{MJ} / \mathrm{kg})$, usando a umidade total na camada, é também utilizado na determinação do grau de carbonificação do carvão. Parâmetros químicos de rank são, ainda, empregados de modo suplementar na caracterização dos depósitos de carvão pela classificação do carvão em camada. A categoria do carvão é definida através da determinação do percentual em massa de cinzas, na base seca. Sendo assim, o uso simultâneo das principais características do carvão, como composição petrográfica, rank e grade, auxiliado por outros parâmetros químicos e físico-químicos, permitiu uma determinação mais acurada do grau de carbonificação.

Considerando a importância científica e comercial da determinação do verdadeiro rank de carvões sul - brasileiros, o objetivo do trabalho é aplicar a mais recente proposta de classificação do carvão, qual seja, a Classificação Internacional do Carvão em Camada, da Comissão Econômica para a Europa das Nações Unidas (CEENU) ao carvão da principal camada (camada Inferior) explotada na Mina do Leão, RS, e verificar os resultados dessa avaliação.

lhos, sendo portadora de importantes depósitos de carvão de idade permiana (Artinskiano-Kunguriano) (Figura 1). Trabalhos anteriores sobre classificação do carvão, segundo o rank, do carvão da Mina do Leão mostram variação de carvão sub-betuminoso $\mathrm{C}$ ao limite entre os estágios carvão sub-betuminoso A/betuminoso alto volátil C, por exemplo, segundo a norma americana American Society for Testing and Materials ASTM D2798 (2011) e.g. Nahuys (1973), Erens (1979) e Corrêa da Silva (1987).

tra amostragem de cerca de $1 \mathrm{~kg}$ foi feita na frente mineira e encaminhada para quarteamento, análises imediatas e petrográficas, no Laboratório de Carvão, UFRGS.

Os valores da umidade (para análise), cinzas, matéria volátil, carbono fixo e da umidade total são expressos em percentual por massa, enquanto os valores do poder calorífico superior, em $\mathrm{MJ} / \mathrm{kg}$. 


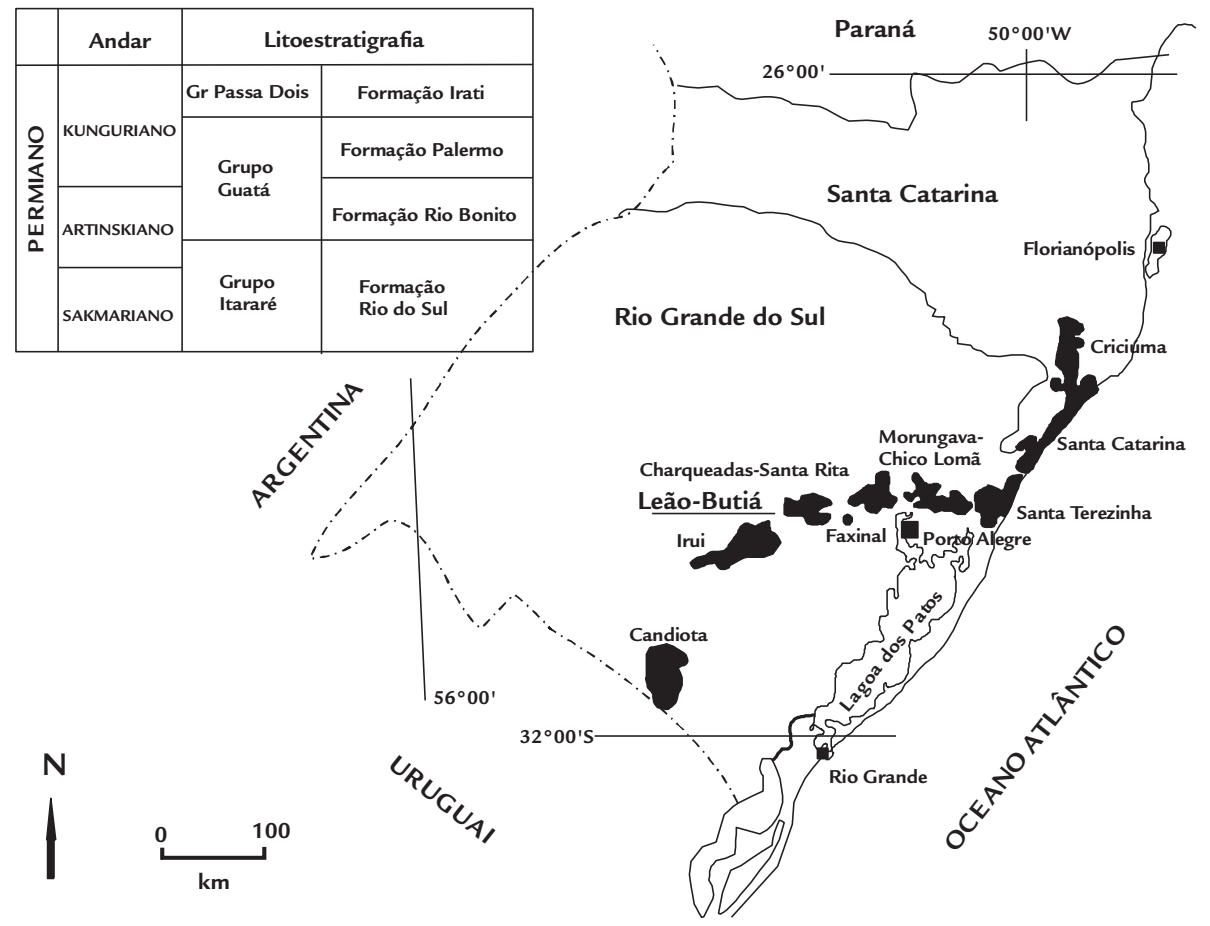

Figura 1

Mapa de localização e coluna estratigráfica da área

(Silva \& Kalkreuth, 2005).

\section{Análises petrográficas}

A análise microscópica das seções polidas foi realizada em microscópio binocular de reflexão LEITZ ORTHOPLANPOL, iluminador POL-OPAK, objetiva de imersão $50 x / 0,85$ oël $\mathrm{P}$, oculares PERIPLAN 10x, com duas fontes de ilumi-

Figura 2

Fotomicrografia ilustrando os grupos Vitrinita, Liptinita e Inertinita

(V, L, I, respectivamente) no carvão da Camada Inferior em luz branca refletida (eixo $\mathrm{x}=200 \mu \mathrm{m})$.

\section{Resultados e discussão}

Os trabalhos sobre a determinação do grau de carbonificação de carvões sul -brasileiros mostram um histórico de dificuldades na determinação do estágio de carbonificação do carvão (rank), através de normas internacionais. Segundo Corrêa da Silva (1990), a variação entre os parâmetros de rank e as medidas do poder refletor da vitrinita está relacionada com a impregnação de substâncias lipídicas na vitrinita, durante o processo de formação do carvão, tendendo a rebaixar os valores. Silva (1994) considera que a intrínseca relação da matéria orgânica com argilo-minerais pode rebaixar os nação: luz branca de $12 \mathrm{~V}$, com potência de 100W; e lâmpada de mercúrio de alta pressão, HBO 100W, para fluorescência. A contagem foi feita com contador de pontos semiautomático SWIFT F 415 C acoplado ao microscópio referido anteriormente. A determinação do poder refletor ( $\mathrm{Ra} \%)$ foi feita em uma seção polida representativa da camada total da Camada Inferior, com determinação de 100 medidas em partículas de colotelinitas, em posição espacial aleatória no mesmo microscópio (Figura 2).

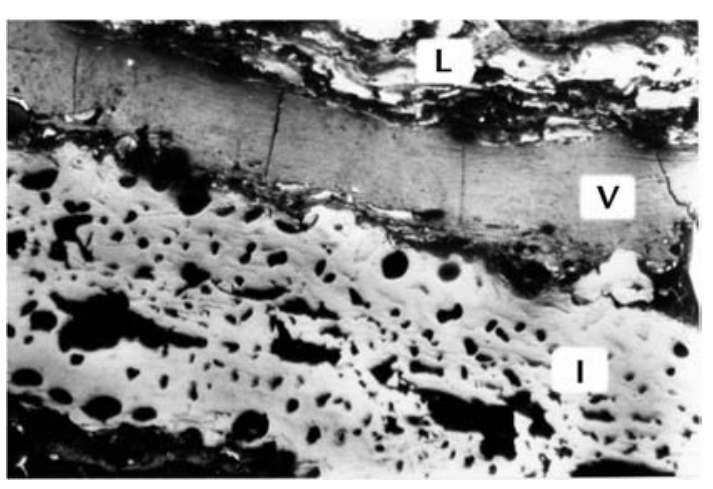

valores do poder refletor aleatório da vitrinita e, por isso, indicar um rank mais baixo.

Em relação ao carvão da Mina do Leão, são raros os trabalhos com enfoque sobre sua classificação segundo o rank e incluem Nahuys (1973), Erens (1979) e Corrêa da Silva (1987). Em estudo preliminar sobre as características petrográficas do carvão da bacia do Leão, Nahuys (1973) utiliza o poder refletor da vitrinita e a percentagem do carbono e classifica o carvão da Mina do Leão no estágio carvão betuminoso alto-volátil, seguindo as normas inglesa e americana. Erens (1979), valendo-se das normas DIN (alemã), não consegue uma classificação acurada do verdadeiro rank do carvão da Mina do Leão e verifica estágios de carbonificação Hartbraunkohle e Flammkoble, o que corresponderia, aproximadamente, à série compreendida entre os estágios carvão sub-betuminoso e carvão betuminoso alto-volátil $\mathrm{B}$, da norma americana (ASTM). Com base na compilação de Nahuys (1973), de Erens (1979), de Corrêa da Silva (1987) e de Silva (1999), observa-se que o poder refletor da vitrinita, um dos parâmetros mais reconhecidamente empregados para a de- 
terminação do rank, não é um parâmetro seguro para ser usado sozinho nessa avaliação. A partir dos dados desses trabalhos, verifica-se que as medidas do poder refletor da vitrinita do carvão da Mina do Leão pode variar de 0,43 até $0,52 \%$. De acordo com esses valores, a classificação do carvão do Leão, segundo o rank, mostra variação na classificação do estágio carvão sub-betuminoso $\mathrm{C}$ até o limite entre os estágios carvão sub-betuminoso A/betuminoso alto-volátil C (ASTM). Esta variação ilustra bem a dificuldade de se avaliar precisamente o rank de carvões sul-brasileiros, quando normas internacionais são empregadas. Essa dificuldade ainda é maior quando se tenta relacionar parâmetros de rank químicos e físicos (Correa da Silva, 1990). Assim, esse trabalho analisa os resultados da aplicação da mais nova proposta internacional de classificação do carvão proposta pela Comunidade Econômica para Europa, Nações Unidas (CEE-NU, 1998), com enfoque na única camada atualmente explotada na Mina do Leão, sul do Brasil. Os dados das análises necessários para essa classificação são discutidos no item 3 de modo pormenorizado e são apresentados na Tabela 1. O método empregado tem a vantagem de usar, de modo simultâneo, os três principais parâmetros empregados na caracterização do carvão (composição, grade e grau de carbonificação ou rank). A seguir, os parâmetros usados e os resultados da aplicação da classificação do carvão em camada, segundo normas da Comissão Econômica para a Europa das Nações Unidas (CEENU, 1998), são apresentados (Tabela 1, Figura 3) e discutidos.

Seguindo as normas da classifica- ção do carvão em camada (CEE - NU 1998), descritas detalhadamente no item 3 , deve-se considerar a regra básica, para a classificação geral do carvão da Camada Inferior e, logo após, deve-se seguir a classificação pormenorizada da referida norma, conforme segue:

1. Regra básica: para aplicação da classificação geral: Grau inferior: poder calorífico superior (base úmida, sem matéria mineral) < $24 \mathrm{MJ} / \mathrm{kg}$ e Ra\% < 0,6.

2. Classificação pormenorizada: quanto à classificação pormenorizada do grau (rank), deve-se atentar para o fato de que essa classificação é feita pelo poder calorífico, uma vez que $\mathrm{Ra} \%$ é inferior a $0,6 \%$. Se $\mathrm{Ra} \%$ for inferior a $0,6 \%$, a classificação deve ser feita, então, pelo poder calorífico.

Seguindo os mesmos critérios das normas de classificação geral e pormeno-

\begin{tabular}{|c|c|}
\hline \multicolumn{2}{|l|}{ Análise petrográfica } \\
\hline Vitrinita \% (sem matéria mineral) & 51 \\
\hline Liptinita \% (sem matéria mineral) & 16 \\
\hline Inertinita \% (sem matéria mineral) & 33 \\
\hline Matéria mineral \% & 15 \\
\hline Poder Refletor da vitrinita \% & $0,41 \pm 0,03$ \\
\hline \multicolumn{2}{|l|}{ Análise imediata } \\
\hline Umidade, U (para análise) \% (SABS 924) & 9,6 \\
\hline Cinzas, C \% (ISO 1171) [bs] & 29,9 \\
\hline Matéria volátil, MV\% (ISO 562) [bsic] & 43,2 \\
\hline Carbono Fixo, CF \% (por diferença) [bsic] & 56,8 \\
\hline \multicolumn{2}{|l|}{ Análise elementar } \\
\hline Enxofre total, S \% (ASTM D4239) [bsic] & 7,08 \\
\hline Carbono, C \% (ASTM D5373) [bsic] & 76,20 \\
\hline Hidrogênio, H \% (ASTM D5373) [bsic] & 4,73 \\
\hline Nitrogênio, N \% (ASTM D5373) [bsic] & 1,36 \\
\hline Oxigênio, O \% (por diferença) [bsic] & 10,63 \\
\hline Poder Calorífico, GCV MJ/kg (ISO 1928) [bsic] & 31,61 \\
\hline FSI (ISO 501) índice de inchamento & 0 \\
\hline Umidade total \% (ISO 5068) & 12,6 \\
\hline Poder Calorífico, GCV MJ/kg [u, sc] & 26,22 \\
\hline
\end{tabular}

rizada referidas anteriormente, o carvão da camada Inferior da Mina do Leão é carvão parabetuminoso ou carvão de grau médio D, de categoria inferior (Figura 3).

Dessa forma, o método aqui aplicado mostra resultados mais satisfatórios do que aqueles anteriormente testados em carvões do sul do país. Isso se deve ao fato de a classificação internacional do carvão em camada considerar, para a classificação do carvão, segundo o rank, os três parâmetros básicos para a caracterização do carvão simultaneamente: composição petrográfica, poder refletor

\section{Conclusões}

Seguindo a classificação empregada, o carvão da camada Inferior, Mina do Leão, é um carvão parabetuminoso (ou de grau médio D) de categoria inferior.

Com base nos resultados obtidos e, comparando-se as classificações internacionais anteriormente aplicadas para a determinação do rank dos carvões sulbrasileiros, a classificação proposta pela Comissão Econômica para a Europa das Nações Unidas mostra-se mais adequa-
Tabela 1

Dados de sete análises do carvão da Camada Inferior, Mina do Leão, RS.

da vitrinita e grade. No caso dos carvões brasileiros, conhecidamente caracterizados por seus elevados conteúdos de minerais (cinzas), a consideração do grade (impurezas), para a determinação da classificação do carvão, permite, certamente, uma avaliação mais acurada.

da, por considerar, simultaneamente, os três principais parâmetros de caracterização do carvão: composição, rank e grade, o que permite uma avaliação mais acurada do estágio e, por conseguinte, na sua utilização. 
Figura 3

Classificação Internacional da Camada Inferior, Mina do Leão, RS, segundo normas CEE, NU (1998).

PCS (h, sc) - poder calorífico superior, base úmida, sem cinzas; L - liptinita, V- vitrinita, I - inertinita, $\mathrm{Ra}$ - poder refletor da vitrinita.

\section{Referências bibliográficas}

ASTM D2798. Standard test method for microscopical determination of the vitrinite reflectance of coal. Annual Book of ASTM Standards, Section 5, Philadelphia, Pa, 2011.

CORRÊA DA SILVA, Z.C. Jazidas de carvão no Rio Grande do Sul, Brasil. In: SIMPÓSIO SUL-BRASILEIRO DE GEOLOGIA, 3. Atas...Curitiba: SBG, 1987. p.677-687.

CORREAA DA SILVA, Z.C. Rank evaluation of south Brazilian Gondwana coals on the basis of different chemical and physical parameters. International Journal of Coal Geology, Amsterdan, n.16, p.209-210, 1990.

BRASIL. Departamento Nacional da Produção Mineral. Companhia de Pesquisa de Recursos Minerais. Projeto Borda leste da Bacia do Paraná. Relatório Interno. Porto Alegre: DNPM/CPRM, 1986.

ERENS, K.H. Petrographische und organischegeochemische Untersuchungen an Koblen aus dem Becken von Leão-Butiá, RS, Brasilien. 1979. 60 f. Diplomarbeit RWTH, Aachen, 1979.

ICCP - International Committee for Coal Petrology. International Handbook of Coal Petrography, 2nd ed. Paris: Centre National de la Recherche Scientifique, 19631971.

MILANI, E. J., FRANÇA, A.B., SCHNEIDER, R.L. Bacia do Paraná. Boletim de Geociências da Petrobras, Rio de Janeiro, n.8, p.69-72, 1994.

NAHUYS, J. Étude préliminaire sur le charbon du Bassin Leão-Butiá. In: CONGRESS INTERNATIONAL GÉOLOGIE STRATIGRAPHIE CARBONIFÈRE, 7. Compte Rendu... Krefeld, 1973, 2, p. 8-21.

SILVA, M.B. Caracterização petrográfica das camadas de carvão da Malha IV, Jazida de Candiota, RS. Porto Alegre: Instituto de Geociências, Universidade Federal do Rio Grande do Sul, 1994. 231 f. (Dissertação de Mestrado em Ciências).

SILVA, M.B. Petrologia de carvões nas jazidas do Leão e Candiota, Permiano Inferior da Bacia do Paraná, RS, Brasil. Porto Alegre: Instituto de Geociências, Universidade Federal do Rio Grande do Sul, 1999. 106 f. (Tese de Doutorado em Ciências).

SILVA, M.B., KALKREUTH, K. Petrological and geochemical characterization of Candiota coal seams, Brazil - implications for coal facies interpretations and coal rank. International Journal of Coal Geology, Amsterdan, n. 64, p.217-238, 2005.

UNITED NATIONS. Economic Comission for Europe. Committee on Sustainable Energy. International Classification of in-seam Coals. New York, 1998. 41 p. (Documento ENERGY, 19). 